number of cell colonies which appear in the cultures. We found that the number of colonies never exceeds 10 even when the viable cell count is high. This may be because the staining test of viability does not reflect the growth potential of the cell or because the non-viable cells present exert some adverse effect on the growth of the viable cells. At present we have not been able to identify which cells do grow, though both fibroblasts and epithelioid cells are present in the cultures; the former are the more vigorous, and they dominate in subcultures.

Adjustment of the total number of cells in the inoculum is desirable as either overcrowded or widely separated cells do not grow rapidly. It is difficult to establish the ideal number, however, as our data do not indicate any clear relation between cell number and success in culture (Table III).

Different methods of setting up the cultures are currently being investigated, since the most crucial stage appears to be the initiation of growth; maintenance of a cell line once established presents much less of a problem.

When it is not possible to obtain amniotic fluid cells for culture, studies may be carried out on cther tissues of fetal origin. In our laboratory randomly selected specimens of amnion have been cultured from explants (Smith et al., 1969) with a $95 \%$ (19 out of 20 specimens) success rate. Similarly cultures of cord explants have also been successful (13 out of 13). Subcultures of amnion and cord have been possible by about the 15th day of culture. Placental tissue has nct been so easy to grow, but no serious attempt was made to overcome the difficulties in view of the ease of cuiture of othcr tissues. Four specimens of chorion all grew within eight days.

It may be that biopsies of fetal skin, membranes, or even fetal blood would be easier material to handle in the laboratory as aids to antenatal diagnosis, but the problems of obtaining these specimens are at present very great.

We should like to express our gratitude to all the obstetricians who kindly sent us specimens of amniotic fluid, particularly Dr. J. G. Robertson, Dr. J. Scrimgeour, and Dr. G. Gordon, and would like to thank Miss E. MacEntire for technical assistance. This work was supported by a grant from the Secretary of State for Scotland.

\section{REFERENCES}

Amarose, A. P., Wallingford, A. J., and Plotz, E. J. (1966). New England fournal of Medicine, 275, 715 .

Chan, W. H., Willis, J., and Woods, J. (1969). fournal of Obstetrics and

Gynaecology of the British Commonwealth, 76, 193.
Emery, A. E. H. (1970). In Modern Trends in Human Genetics. London, Butterworths. In press.

Gordon, H., and Brosens, I. (1966). Obstetrics and Gynecology, 30, 652.

Jacobson, C. B., and Barter, R. H. (1967). American fournal of Obstetrics and Gynecology, 99, 795.

Klinger, H. P., and Miller, O. J. (1968). In Diagnosis and Treatment of Fetal Disorders, p. 72. New York, Springer.

Nadler, H. L. (1968). Pediatrics, 42, 912.

Nadler, H. L., and Gerbie, A. B. (1969). American fournal of Obstetrics and Gynecology, 103, 710

Smith, M., MacNab, J., and Ferguson-Smith, M. A. (1969). Obstetrics and Gynecology, 33, 313.

Steele, M. W., and Breg, W. R., jun. (1966), Lancet, 1, 383.

Thiede, H. A., Creasman, W. T., and Metcalfe, S. (1966). American fournal of Obstetrics and Gynecology, 94, 589.

Valenti, C., and Kehaty, T. (1969). Fournal of Laboratory and Clinical Medicine, 73, 355 .

Votta, R. A., Bobrow de Gagneten, C., Parada, O., and Giulietti, M. (1968). American fournal of Obstetrics and Gynecology, 102, 571.

Wachtel, E., Gordon, H., and Olsen, E. (1969). Fournal of Obstetrics and Gynaecology of the British Commonwealth, 76, 596.

\title{
Assessment of Paracervical Nerve Block Anaesthesia during Labour
}

\author{
P. J. MURPHY, ${ }^{*}$ M.B., M.A.o., M.R.c.o.G. ; J. D. WRIGHT, $\dagger$ M.B., M.R.c.o.G., \\ T. B. FITZGERALD, $\ddagger$ M.B., F.R.C.S.ED., F.R.C.o.G.
}

\begin{abstract}
Cummary: An assessment of paracervical nerve tlock anaesthesia was made in 118 women during labour using $0.5 \%$ bupivacaine with 1:200,000 parts of adrenaline added. The 82 multiparae were each given a single block and the 36 primigravidae a continuous block to try to provide the sole means of analgesia throughout the first stage of labour.

Only $48 \%$ of the primigravidae required no other form of analgesia, as against $72 \%$ of the multiparae.

Complications included a $17 \%$ incidence of maternal haemorrhage during insertion of the continuous needle, an overall fetal bradycardia rate of $11 \%$, and two perinatal deaths following single paracervical block. It is concluded that the use of continuous paracervical block should be discontinued, and that amide-type local anaesthetic agents should not be used for single paracervical block.
\end{abstract}

*Clinical Tutor.

tResidential Surgical Officer.

St. Mary's Hospitals for Women and Children, Manchester 13.

\section{Introduction}

The use of paracervical nerve block anaesthesia to provide analgesia in labour was first described by Gellert (1926). Since then several workers have provided evidence of its efficacy (Freeman et al., 1956; Davis et al., 1962; Cooper and Moir, 1963). A wider usage in obstetric practice might have been expected but for the short duration of analgesia provided by the local anaesthetic agents available.

Two methods of increasing the duration of analgesia without the need for repeated vaginal puncture have been reported. Firstly, the recently introduced local anaesthetic agent bupivacaine has been shown to provide analgesia for three to four hours after a single paracervical nerve block (Kuah and Yates, 1967; Cooper et al., 1968; Gudgeon, 1968; Picton 1969). Secondly, the use of continuous block which was described by Tafeen et al. (1966). This enables repeated injections of local anaesthetic agent to be administered through fine precurved polyvinyl catheters inserted and retained in the paracervical spaces.

The purpose of this study was to determine whether bupivacaine could be used to provide the sole means of 
safe analgesia throughout the first stage of labour, when administered to multiparae by single block and to primigravidae by continuous block.

\section{Patients Studied}

The patients were carefully selected and only those without evidence of a hypertensive state, placental insufficiency, or disproportion were included. This left a total of 118 patients (82 multiparae and 36 primigravidae). Labour occurred spontaneously in most patients, but surgical induction was performed in some. The oxytocin drip was not used to induce or accelerate labour.

A paracervical block was administered to each patient when labour was established and analgesia was thought necessary. The technique of single block used in the multiparae has been described by Cooper and Moir (1963). Continuous block was administered to the primigravidae by the method described by Tafeen et al. (1966). The instruments are shown in Figs. 1 and 2. The pointed

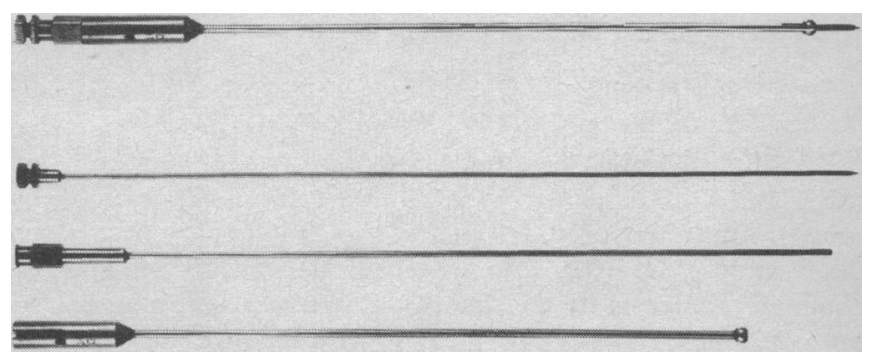

FIG. 1.-Puncture needle with inner and outer guide tubes.

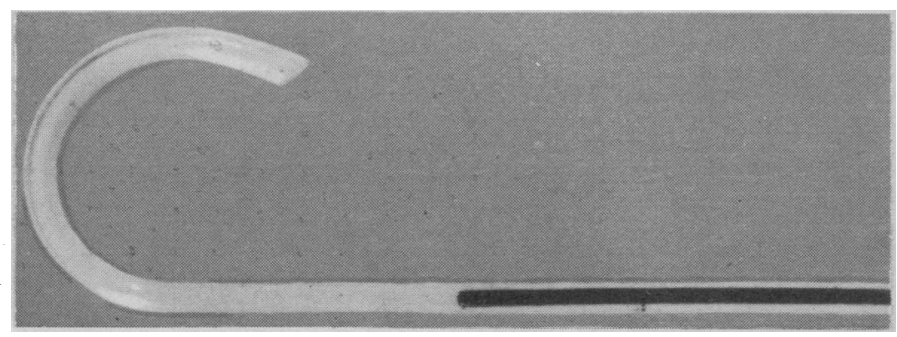

FIG. 2.-Distal portion of a precurved catheter (enlarged).

puncture needle and guide tubes are seen in assembled and component form (Fig. 1.) The distal third of a precurved polyvinyl catheter is shown with the wire stylet inserted (Fig. 2). Before introducing the catheter into the paracervical space the precurved end is straightened by inserting the wire stylet to the tip of the catheter.

The assembled puncture needle and guide tubes were inserted into the vagina, and the puncture needle and inner guide were pressed through the lateral fornices at 9 o'clock on the right and at 3 o'clock on the left. The puncture needle was withdrawn and the site aspirated with a syringe attached to the inner guide tube to exclude accidental puncture of the blood vessels. The paracervical space was then distended with $2 \mathrm{ml}$. of normal saline. The precurved catheter, straightened by the wire stylet, was passed through the inner guide tube and into the paracervical space, the required depth of insertion being indicated by a mark on the wire stylet. The catheter stylet was withdrawn, allowing the precurved portion to re-form and be retained in the paracervical space. The guide tubes were withdrawn over the catheter, and a catheter obturator with plastic closing cap was inserted into the proximal end of the catheter to prevent reflux. The end of the catheter was attached to the patient's lower abdomen with adhesive tape.

All injections of anaesthetic agent were given through these catheters, and before injections were repeated the siting of the catheters was checked by vaginal examination.

The local anaesthetic agent used throughout was bupivacaine (1- $n$-butyl-DL-piperidine-2 carboxylic acid-2:6dimethylanilide hydrochloride) 0.5 solution with $1: 200,000$ adrenaline solution added. Ten millilitres of anaesthetic solution was injected on each side at both single and continuous paracervical nerve block. The analgesia obtained after block was assessed subjectively and was taken to mean the absence of both sacral and lower abdominal pain during a normal contraction.

Any variation from this standard of analgesia was regarded as evidence of failure of the technique. The maternal blood pressure and pulse and the fetal heart rate were recorded at 15-minute intervals after every block.

The onset of the second stage of labour was signalled by the desire to bear down or by rectal discomfort. If the analgesic effect of the block wore off before these signs were evident a vaginal examination was made to assess the cervical dilatation and the need for further analgesia.

In primigravid patients if the cervix was not fully dilated a further injection was made by means of the selfretaining catheters, but if the previous injection had been given within one hour the technique was abandoned as a failure and systemic analgesia was substituted. If the patient was fully dilated the catheters were removed. In the multiparous patients if the analgesic effect of the single block wore off before full dilatation was achieved alternative analgesia was provided.

\section{Continuous Paracervical Block in 36 Primigravidae}

Analgesia Throughout Labour.-In 13 patients successful analgesia was provided throughout the first stage of labour. Two patients became fully dilated during the analgesic effect of the first injection and were delivered three to four hours after the injection (Table I). Eleven patients received a second

Table I.-Duration of Analgesia in 13 Patients After the Initial Injection

\begin{tabular}{lcccccc|c|c|c}
\hline Hours &. &. &. &. &. &. & $1-$ & $2-$ & $3-$ \\
No. of patients &. &.. &.. &.. &.. & 2 & 3 & 8 \\
\hline
\end{tabular}

injection, six becoming fully dilated during the analgesic effect (Table II). These six patients were delivered two to four hours after the second injection. The remaining five received a

TABle II.-Duration of Analgesia in 11 Patients After the Second Injection

\begin{tabular}{|c|c|c|c|c|c|c|c|c|}
\hline Hours & . & . & . & . & $\ldots$ & $1-$ & $2-$ & $3-$ \\
\hline No. of patients & $\ldots$ & .. & .. & .. & .. & 2 & 6 & 3 \\
\hline
\end{tabular}

third injection, and all became fully dilated during the analgesic effect. Delivery in these five took place one to three hours after the third injection. The duration of labour in the 13 patients was 6 to 14 hours. All the deliveries were normal and the babies were healthy.

Initial Analgesia Only. - The method provided analgesia after the initial injection in 11 patients but was considered to have failed in two in which analgesia lasted for less than one hour (Table III). The remaining nine each re- 
TABle III.-Duration of Analgesia in 11 Patients After the Initial Injection

\begin{tabular}{lllll|l|l|l|l}
\hline Hours & $\ldots$ & $\ldots$ & $\ldots$ & $\ldots$ & $0-$ & $1-$ & $2-$ & $3-$ \\
No. of patients & $\ldots$ & $\ldots$ & $\ldots$ & 2 & 5 & 3 & 1 \\
\hline
\end{tabular}

ceived a second injection; five of them noted no analgesic effect and four obtained analgesia for less than $30 \mathrm{~min}-$ utes. In all nine cases the method was thought to have failed. The duration of labour in the 11 patients was 7 to 12 hours. Ten were delivered normally and one required a forceps extraction. All the babies were healthy at delivery.

No Analgesia Obtained.-The method failed to provide analgesia in three patients who received the initial injection.

Method Abandoned Owing to Complications.-The following complications arose in nine patients and resulted in the method being abandoned. (1) Haemorrhage: In six patients haemorrhage occurred after insertion of the puncture needle and guide tube into the paracervical space. The haemorrhage was noted during the routine aspiration of the site. The method was immediately abandoned in these cases. The haemorrhage did not persist after withdrawal of the puncture needle and guide tube, and there was no evidence of parametrial haematoma formation during the puerperium. (2) Fetal bradycardia: This was detected in three patients 10 to 15 minutes after the initial injection. In two the fetal heart rate decreased to $100-110$ beats a minute, and in the third a decrease to 90 beats a minute was noted. A gradual increase in rate occurred in all three cases, and after one hour normal fetal heart rates were recorded. No further fetal heart irregularities were noted in these labours. All the babies were delivered normally and appeared healthy at birth. The catheters were withdrawn and the technique was abandoned in each case when fetal bradycardia was detected.

\section{Single Paracervical Block in 82 Multiparae}

Fifty-nine patients obtained analgesia from the single paracervical nerve block and reached the second stage of labour without any other form of analgesia. The interval between insertion of the block and onset of the second stage in these patients is shown in Table IV. In 16

Table IV.-Duration of Analgesia in 59 Patients After Single Paracervical Block

\begin{tabular}{llll|l|l|l|l|l}
\hline Hours &. & $\ldots$ & $\ldots$ & $1-$ & $2-$ & 3 & $4-$ & $5-$ \\
No. of patients & $\ldots$ & $\ldots$ & 2 & 4 & 20 & 25 & 8 \\
\hline
\end{tabular}

patients the analgesic effect ceased before the beginning of the second stage and in seven no analgesia was obtained. The following complications were found.

\section{Fetal Bradycardia}

A fetal heart rate of less than 110 was recorded in nine patients. In eight of these bradycardia occurred 10 to 20 minutes after the block, but a return to normal was noted after a further 30 minutes. All were delivered normally and the babies appeared healthy at birth. In the ninth patient the bradycardia persisted and was followed by death of the fetus.

\section{Perinatal Death}

Two stillbirths occurred after paracervical block and the cases are reported in detail.

Case 1.-This patient, a para-1 aged 23, was booked for hospital confinement. The antenatal period was uneventful and labour was induced before term because of a breech presentation. A surgical induction was performed and labour became established two and a half hours later. With the cervix three fingerbreadths dilated a block was administered. The fetal heart rate was 140 immediately following the block, but within 15 minutes fetal bradycardia was noted and 30 minutes after the block the rate had fallen to 80 Five minutes later the fetal heart was no longer audible. The patient felt nauseated and had vomited 30 minutes after the block; she had obtained only 30 minutes' analgesia. Eventually a stillborn female infant was delivered; it weighed 3,200 g., and the placenta $397 \mathrm{~g}$. At necropsy petechial haemorrhages were noted over the brain surface and a small cerebral haemorrhage was seen. Analysis of a sample of fetal lung showed a bupivacaine concentration of $6.89 \mu \mathrm{g} . / \mathrm{g}$. of tissue.

Case 2.-This patient, a para-1 aged 18, was booked for hospital confinement. The antenatal period was uneventful and the onset of labour was spontaneous at term. When labour was established a paracervical block was administered but no analgesia was obtained. The patient experienced a mild rigor 30 minutes after the block had been given. The fetal heart rate was normal after the block and was checked every 15 minutes as labour proceeded and found to be normal. Four hours after the block routine auscultation revealed that the fetal heart beat was absent. One hour later a stillborn female infant weighing 3,670 g. was delivered together with a placenta weighing $450 \mathrm{~g}$. Necropsy revealed only petechial haemorrhages over the brain surface. Analysis of a sample of fetal lung showed a bupivacaine concentration of $4 \cdot 37 \mu \mathrm{g} . / \mathrm{g}$. of tissue. No maternal complications occurred.

\section{Discussion}

In this investigation the $0.5 \%$ bupivacaine solution was used in preference to the $0.25 \%$ solution, which had been found to provide ineffective analgesia in a small pilot series. Of the 27 uncomplicated primigravidae, 24 obtained analgesia from the initial paracervical nerve block but only $13(48 \%)$ obtained a continuance of analgesia during the first stage of labour following repeated injections. Nine patients obtained little or no analgesia from the second injection, and since the site of the injection and the dose of bupivacaine were similar at each injection, failure to achieve further analgesia could be attributed to tachyphylaxis.

Of the 82 multiparae, 75 benefited from the single block, and $59(72 \%)$ of these required no other form of analgesia throughout the first stage of labour.

These results suggest that paracervical nerve block would be more useful in multiparous labour in which the duration of analgesia required and that provided by the bupivacaine are more appropriate.

Maternal haemorrhage was seen in six patients during the insertion of the continuous block puncture needle and inner guide tube into the paracervical space. The incidence was unexpectedly high and had not been reported previously. Puncture of the vessels at the base of the broad ligament may be explained by the observation that the puncture needle and inner guide tube used in this series protruded $13 \mathrm{~mm}$. beyond the outer guide tube. Cooper and Moir (1963) and Davis et al. (1962) suggested that a needle should protrude no more than $7 \mathrm{~mm}$. if puncture of the vessels is to be avoided. Nevertheless, Freeman et al. (1956) used a needle which protruded 15 $\mathrm{mm}$. and reported no maternal haemorrhage in their series. In the present series the frequent occurrence of maternal haemorrhage showed the technique to be unsafe in the form used.

Fetal bradycardia occurred in both groups, the overall rate for the series being $11 \%$. Fetal bradycardia following paracervical block with $0.5 \%$ bupivacaine with or without adrenaline has been reported by several workers (Stockhausen, 1967; Cooper et al., 1968; Hollmén et al., 1969; Jung et al., 1969; Teramo, 1969). The significance of fetal bradycardia after paracervical block has not been appre- 
ciated in the past and Gordon (1968) stated that it occurs as a result of the direct toxic action of the local anaesthetic agent on the fetal myocardium and is not a sign of fetal asphyxia. Teramo (1969) found a correlation between fetal bradycardia and a decrease in the fetal $p H$ after paracervical block; he concluded that fetal bradycardia after paracervical block is sufficient to cause hypoxia. Two perinatal deaths occurred after paracervical block, both babies being stillborn.

At necropsy both babies were shown to have fetal tissue levels of bupivacaine about 10 times the expected normal levels, indicating massive accumulation in the fetal circulation (F. Reynolds, personal communication, 1969). Gordon (1968) showed that after a paracervical block the local anaesthetic agent can be absorbed from the paracervical tissues and that it reaches a peak concentration in the maternal circulation after 20 minutes; after crossing the placenta the peak fetal concentration is reached 30 minutes after the block. Shnider et al. (1968) considered that some of the local anaesthetic agent could reach the placenta direct from the paracervical tissues. Page et al. (1961) and Teramo and Widholm (1967) noted the possibility of accidental injection of local anaesthetic agent direct into the fetus during insertion of the paracervical block. The high fetal accumulation of bupivacaine in the two stillbirths could have occurred by any of these routes.

The first fetal death followed the accepted pattern (Whitehouse, 1968), but the second is difficult to explain. It is suggested that the accumulation of bupivacaine may have occurred more slowly in the second fetus and that placental vasoconstriction and a decrease in placental blood flow took place. Teramo and Widholm (1967) postulated that mepivacaine may interfere with placental blood flow and cause vasoconstriction. Rosefsky and Petersiel (1968) showed that the fetus is unable to metabolize local anaesthetic agent and is dependent on the placenta for its removal. Thus bupivacaine may have accumulated and been retained in the fetus and as labour progressed an increase in fetal acidosis could be expected. In the presence of acidosis the cardiotoxic effects of bupivacaine would be potentiated and could have resulted in acute fetal cardiac failure (Steinhaus, 1957).
In this assessment of paracervical block for routine use in labour we have found the following disadvantages: (a) a low rate of success with continuous block, (b) a high incidence of maternal haemorrhage during insertion of the continuous block needle, and (c) a high incidence of fetal bradycardia together with two perinatal deaths.

In conclusion, we recommend that continuous block in the present form should be discontinued and that the amide-type local anaesthetic agents, of which bupivacaine is one, should not be used for single paracervical nerve block.

We wish to thank Dr. R. Ollerenshaw, department of medical illustration, Manchester Royal Infirmary, for his help with the illustrations. We are indebted to Dr. Felicity Reynolds, department of pharmacology and therapeutics, St. Thomas's Hospital Medical School, London, for estimating the tissue levels of bupivacaine in the two stillbirths and for her helpful comments.

\section{REFERENCES}

Cooper, K. V., and Moir, J. C. (1963). British Medical fournal, 1, 1372. Cooper, K. V., Gilroy, K. J., and Hurry, D. J. (1968). Fournal of Obstetrics and Gynaecology of the British Commonwealth, 75, 863. Davis, J. E., Frudenfeld, J. C., Frudenfeld, K., and Webb, A. N. (1962). Obstetrics and Gynecology, 19, 195.

Freeman, D. W., Bellville, T. P., and Barno, A. (1956). Obstetrics and Gynecology, 8, 270.

Gellert, P. (1926). Monatsschrift für Geburtshilfe and Gynäkologie, 73, 143.

Gordon, H. R. (1968). New England Fournal of Medicine, 279, 910.

Gudgeon, D. H. (1968). British Medical fournal, 2, 403.

Hollmén, A., Oiala, A., and Korhonen, M. (1969). Acta Anaesthesiologica Scandinavica, 13, 1 .

Jung, H., Konecky, P., and Klöck, F. K. (1969). Geburtshilfe und Frauenheilkunde, 29, 174.

Kuah, K. B., and Yates, M. J. (1967). Lancet, 1, 1159. Kuah, K. B., and Yates, M. J. (1967). Lancet, 1, 1159.

fournal of Obstetrics and Gynecology, 81, 1094.
Picton, F. C. R. (1969). British fournal of Clinical Practice, 23, 162.

Rosefsky, J. B., and Petersiel, M. E. (1968). New England fournal of Medicine, 278, 530 .

Shnider, S. M., Asling, J. P., Margolis, A. J., Wey, E. L., and Wilkinson, G. R.'(1968). New England fournal of Medicine, 279, 947.

Steinhaus, J. E. (1957). Anesthesiology, 18, 275.

Stockhausen, H. (1967). Deutsche medizinische Wochenschrift, 92, 2220.

Tafeen, C. H., Freedman, H. L., and Harris, H. (1966). American fournal of Obstetrics and Gynecology, 100, 55 .

Teramo, K. (1969). Fournal of Obstetrics and Gynaecology of the British Commonwealth, 76, 881.

Teramo, K., and Widholm, O. (1967). Acta Obstetricia et Gynecologica Scandinavica, 46, Suppl. No. 2.

Whitehouse, D. B. (1968). British Medical fournal, 2, 764.

\title{
Plasma Magnesium Concentration in Primary Hyperparathyroidism
}

\author{
R. A. L. SUTTON, ${ }^{*}$ B.M., M.R.C.P.
}

\begin{abstract}
ummary : The plasma magnesium concentration has $\checkmark$ been determined in 73 patients with primary hyperparathyroidism. In most patients it lay within the normal range $(1.7-2.3 \mathrm{mg} . / 100 \mathrm{ml}$.$) , but in five it was less than$ $1.6 \mathrm{mg} . / 100 \mathrm{ml}$. These patients had relatively high urinary magnesium outputs, and one of them, studied in greater detail, failed to retain parenterally administered magnesium. Hence hypomagnesaemia in hyperparathyroidism may be associated with a defect in renal magnesium conservation, which may be reversible.
\end{abstract}

* Senior Medical Registrar, University College Hospital, London W.C.1.
The parathyroid glands have for many years been known to
influence magnesium metabolism. Bulger and Gausmann (1933)
noted that a negative magnesium balance accompanied primary
hyperparathyroidism with osteitis fibrosa; they and many sub-
sequent workers observed a positive magnesium balance, asso-
ciated with a fall in the plasma magnesium concentration, after parathyroidectomy in such patients. Parathyroid hormone has been shown to increase renal tubular magnesium reabsorption in rats (MacIntyre et al., 1963) and in man (Shelp et al., 1966). MacIntyre et al. (1963) postulated a central role for the para- 